\title{
EVOLUCIÓN DE LAS DISPARIDADES ESPACIALES DEL BIENESTAR EN ESPAÑA, 1860-1930. EL ÍNDICE FÍSICO DE CALIDAD DE VIDA
}

\author{
RAFAEL DOMÍNGUEZ MARTÍN \\ y MARTA GUIJARRO GARVI \\ Universidad de Cantabria
}

\section{RESUMEN}

En este trabajo se plantea la construcción del Índice Físico de Calidad de Vida (IFCV) a partir de variables demográficas y educativas, para las regiones y provincias españolas en el contexto europeo, entre 1860 y 1930. Los resultados obtenidos indican que en ese período se produjo una mejora, desigual, pero generalizada, del IFCV y superior a la experimentada en términos de producto per capita. Por otro lado, la evolución de las disparidades espaciales del IFCV sugiere algunos paralelismos con el caso italiano y también la necesidad de tener una visión más cautelosa de la relación entre crecimiento económico y bienestar.

\begin{abstract}
In this article we propose the construction of Physical Quality of Life Index (PQLI) from demographical and educative variables, applied to Spanish regions and provinces in the European context between 1860 and 1930 . Our results show an unequal but extensive improvement of PQLI, which was superior to the one experimented in per capita product in this period. Morover, the evolution of PQLI spacial disparities suggests some parallels with Italian case and the necessity to be careful about the relationships between economic growth and well-being.
\end{abstract}




\section{INTRODUCCIÓN ${ }^{1}$}

En los últimos años se ha empezado a tomar conciencia de la necesidad de cambiar el énfasis que la historia económica había puesto en el crecimiento en detrimento de los aspectos relacionados con el bienestar y la calidad de vida, aspectos que la economía del desarrollo venía estudiando con creciente atención desde la década de los sesenta y que sólo muy lentamente se han abierto camino en nuestra disciplina ${ }^{2}$. Este trabajo incorpora uno de esos instrumentos, el Índice Físico de Calidad de Vida de Morris (1979), y tiene por objeto evaluar las disparidades y tendencias del bienestar en España a escala provincial y regional en un período clave de la modernización económica del país.

La existencia de acusados contrastes espaciales en el bienestar generado por el crecimiento económico - y su tendencia a aumentar hasta un determinado momento - es una característica típica de los países atrasados, pero la contabilidad nacional es incapaz de reflejarla convenientemente para épocas históricas como la estudiada, máxime en el caso de las economías que se encuentran en los primeros estadios del desarrollo. En esa situación, una parte sustancial del consumo que satisface necesidades básicas no se realizaba a través del mercado, de manera que los ámbitos territoriales más mercantilizados podrían presentar unas diferencias en niveles de vida con respecto a los menos integrados mercantilmente no correspondientes con las diferencias reales en términos de bienestar.

Para soslayar estos problemas nos proponemos partir de la distinción entre medios (el ingreso per capita) y fines (el bienestar), distinción que tiene una larga y prestigiosa genealogía intelectual en la historia del pen-

1 Este trabajo se ha realizado con financiación del proyecto de la DGICYT PB93-0215-01. Agradecemos especialmente a Roser Nicolau algunas pistas para conseguir información, así como el envío diligente de varios trabajos de difícil acceso. Fausto Dopico nos facilitó los datos de esperanza de vida sin todavía estar publicados en el futuro libro que, junto con David-Sven Reher, ha escrito sobre El declive de la mortalidad en España. Aida Ramos, con su increible paciencia, nos ayudó en la confección de los mapas. Finalmente, las críticas de los evaluadores anónimos sirvieron para aclarar nuestros propios puntos de vista y para mejorar el producto final del cual sólo nosotros somos responsables.

2 Véase Othick (1983). Zamagni (1989, p. 125) ha sugerido utilizar «más extensa y sistemáticamente» indicadores de calidad de vida como la esperanza de vida, la mortalidad infantil y la alfabetización. Fontana (1990, p. 25) ha llamado a incorporar los conceptos de Sen para el estudio de la calidad de vida. Y Crafts (1993 y 1997) ha evaluado los niveles de vida en Gran Bretaña en comparación con otros países europeos, incorporando indicadores de seguridad, salud, ocio y calidad medioambiental para el período 1950-1989, y sendas reconstrucciones del Indice de Desarrollo Humano y del indicador de Dasgupta y Weale (1992) para la época de la revolución industrial. 
samiento económico, y que ha sido revivida en los últimos tiempos por Amartya Kumar Sen, cuyas hipótesis y conceptos se analizan en la primera parte. En la segunda, se plantean las limitaciones y metodología para la construcción del Índice Físico de Calidad de Vida (IFCV), una de las medidas más útiles del bienestar en términos senianos, del cual se presenta una primera foto fija para las regiones españolas en el contexto europeo (con especial referencia a Italia). En el tercer apartado se resumen los resultados obtenidos en la construcción del IFCV a nivel provincial y regional: el aumento de la dispersión territorial del bienestar que acompañó a los primeros momentos del crecimiento económico moderno en España no fue impedimento para que se produjera una mejora, desigual pero generalizada, del IFCV (y superior a la experimentada en términos de producto per capita); hacia 1930, la correlación entre el ranking de IFCV a nivel regional, por un lado, y la estructura de la propiedad de la tierra y la productividad del trabajo agrícola y los rendimientos por unidad de superficie, por otro, arrojan unos valores muy significativos; finalmente, la evolución de las disparidades espaciales del bienestar y el ranking regional del IFCV presentan importantes diferencias en comparación con la evolución de las disparidades de renta regional per capita y el ranking de la misma en el período 1860-1930.

\section{CONTABILIDAD NACIONAL Y MEDICIÓN DEL BIENESTAR}

En el período que se considera en esta investigación, España era una economía atrasada que había iniciado su transición al desarrollo, con un notable dominio de la producción y el empleo agrarios, y con una productividad relativa de este sector muy alejada de la productividad media de la economía, que además tendió a ensancharse hasta la I Guerra Mundial. Como consecuencia de ello, las estimaciones del PIB, al igual que en el caso de Italia o Rusia, resultan muy deficientes ${ }^{3}$ y -lo que es más importante- escasamente significativas para medir el nivel de vida a nivel regional, debido a las enormes disparidades espaciales en la distribución del producto per capita y la aportación al bienestar de toda una serie de bienes y servicios que no pasaban por el mercado. Según Williamson (1965

${ }^{3}$ Algunas evaluaciones muy críticas a las estimaciones históricas del PIB en España se pueden ver en Bustelo (1993, pp. 159-173) y Nadal y Sudriá (1993, pp. 199-204). Carreras, por su parte, ha realizado una valoración más constructiva (Bardini, Carreras y Lains, 1995, pp. 124-132). 
y 1981), la distribución espacial de la renta en esos primeros momentos de transición al desarrollo se torna más desigual, puesto que el sector agrario de baja productividad presenta una localización dispersa, frente a la localización concentrada de la actividad industrial de mayor productividad relativa ${ }^{4}$. En consecuencia, en los países atrasados, como lo era España en el período considerado, hay una justificación adicional para buscar indicadores alternativos que nos ayuden a medir las disparidades territoriales y sus tendencias en la consecución de unos niveles de bienestar aceptables para la mayoría de la población.

En este sentido, los trabajos teóricos de Sen tienen un enorme interés para los historiadores. Según este autor, la economía del desarrollo debe abandonar el énfasis en el ingreso agregado y tomar en consideración «los "derechos de acceso" (entitlements) de la gente y las "capacidades" (capabilities) que esos derechos generan». Frente a las libertades negativas (las libertades políticas), los entitlements son los derechos positivos («libertades positivas») a la salud y a la educación, mientras que

«las capacidades de las personas dependen, entre otras cosas, de los grupos de mercancías de los cuales pueden disponer. En cada sociedad existen reglas que gobiernan quién puede hacer uso de qué, y la gente persigue sus objetivos sujeta a estas reglas... El conjunto de todos los grupos de mercancías de los cuales una persona puede elegir uno se puede denominar "derecho de acceso" de la persona».

Sen dintingue también entre capacidades y consecuciones (functionings). Los functionings son «características personales; nos dicen lo que una persona está haciendo», en cambio «la capacidad para funcionar refleja lo que una persona puede hacer». Dado que las «mercancías no son más que medios para otros fines» y que el PNB, como conjunto de mercancías, es sólo «una medida de los medios de bienestar que tiene la gente y no nos dice nada de lo que la gente en cuestión conseguirá en el futuro con estos medios, dados estos fines», la noción de capacidad (para funcionar) resulta mucho más próxima a la de nivel de vida que otros conceptos

${ }^{4} \mathrm{El}$ argumento termina con que luego los beneficios del crecimiento tienden a difundirse espacialmente y se produce una progresiva equiparación espacial de la renta. Esta nueva curva en $U$ invertida de la desigualdad regional presenta, no obstante, algunas excepciones. En Francia, según el análisis a largo plazo de Toutain (1981, pp. 307-311), cambió la tendencia a la equiparación regional de la renta, que al menos se había iniciado desde 1840 , en algún momento en el que aumentaron las disparidades regionales entre 1930 y 1970. En el caso de España, Prados (1992, p. 34) muestra un descenso de las disparidades entre 1900 y 1930 , seguido de un nuevo aumento hasta 1950. 
derivados de la economía del bienestar como utilidad y posesión, que no son partes constituyentes del nivel de vida. En concreto, el bienestar (well-being) de una persona depende de las «consecuciones alcanzadas por esa persona», entendiendo por tales la «habilidad para hacer ciertas cosas y para conseguir ciertos tipos de estados (beings) (tales como el estado de buena nutrición, el estado de liberación de la morbilidad evitable, el estado de capacidad de desplazarse como se desee, y así sucesivamente)». Las functionings relevantes pueden variar desde las cosas elementales (como las anteriores) a «consecuciones más complejas, como el estado de felicidad, el alcance de la realización personal, la participación en la vida de la comunidad, y así sucesivamente» ${ }^{5}$.

Este tipo de planteamiento que tiene una ilustre genealogía intelectual en la historia del pensamiento económico, inspiró directamente la construcción del Índice de Desarrollo Humano (IDH) del Programa de Naciones Unidas para el Desarrollo (PNUD) y responde básicamente a la filosofía del IFCV, auspiciado por el Overseas Development Council de Washington. El IFCV, elaborado por Morris (1979), buscaba comparar el bienestar de los países desarrollados y los subdesarrollados a partir de tres indicadores (la esperanza de vida a la edad de un año, la mortalidad infantil y la tasa de alfabetización adulta) mediante la construcción de un índice compuesto simple ${ }^{6}$. Aunque el estudio de Morris (1979) constataba que los países con un PNB per capita bajo solían tener unos IFCV reducidos, y, al contrario, los países de elevado PNB per capita tendían a presentar unos IFCV altos, la elevada correlación estadística entre ambos indicadores $(r=0,79)$ ocultaba importantes asimetrías: en algunos casos había una relación asimétrica entre PNB per capita e IFCV, lo que sugiere que se podían conseguir mejoras importantes en la calidad de vida antes de lograr algún incremento del PNB per capita (caso de Sri Lanka); y, a la inversa, que un nivel elevado de PNB per capita no garantizaba necesariamente una mejor calidad de vida (caso de algunos miembros de la OPEP).

${ }^{5}$ Sen $(1984$, pp. $315-317,334,497-499,511-516 ; 1987$, p. $16 ; 1988$, p. $15 ; 1995$, p. 39). Para ulteriores especificaciones de los términos capabilities y functionings ver Sen (1993, pp. 30-31, 38).

- Para cada indicador, el nivel de cada país se valoraba en una escala de 0 a 100 , donde 0 representa el peor nivel y 100 el mejor en esperanza de vida y al contrario en mortalidad infantil. Dado que el último indicador, la tasa de alfabetización, se mide en porcentajes, la escala venía dada directamente (obsérvese que tal procedimiento altera después los, ya de por sí arbitrarios, criterios de ponderación, pues aunque en la muestra de Morris aparecían países con tasas de alfabetización del 100 por 100 , no había en cambio ninguno con 0 por 100). Una vez que los niveles de un país estuvieran representados en la escala 0 a 100 , el IFCV se calculaba mediante la media aritmética de los tres. 


\section{EL IFCV: LIMITACIONES, CONSTRUCCIÓN Y APLICACIÓN AL CASO ESPAÑOL}

El IFCV tiene algunas limitaciones importantes, como una definición muy estrecha de «calidad de vida», un criterio de ponderación arbitrario y aplicado además con incoherencia (al mantenerse al margen la escala de la alfabetización), la correlación existente entre algunas variables (en este caso, entre la esperanza de vida y la mortalidad infantil, máxime teniendo en cuenta los altos índices de mortalidad infantil) y la no consideración del retardo temporal con que opera sobre el nivel de vida el proceso de alfabetización ${ }^{7}$. Pero no es teóricamente anodino: contiene una función de bienestar implícita al definir el bienestar como la capacidad de disfrutar de una larga vida con la habilidad para comunicarse y aumentar el conocimiento. Ello le hace especialmente útil para el estudio de las economías atrasadas del pasado, en la medida en que incorpora directamente las consideraciones relativas al bienestar en términos de resultados materiales para la salud y la educación de la población, desplazando el énfasis del Sistema de Cuentas Nacionales en el consumo, una variable mucho más importante en etapas posteriores del crecimiento económico. $\mathrm{Y}$, además de ser preferible a otros indicadores sociales alternativos que intentan medir el nivel de desarrollo a través de sus cambios estructurales, el IFCV presenta dos importantes ventajas añadidas.

En primer lugar, el IFCV incluye los componentes (esperanza de vida, mortalidad infantil y alfabetización) que son - según la mayoría de los expertos- más relevantes para medir el bienestar en situaciones de baja renta per capita ${ }^{8}$. La segunda ventaja añadida del IFCV es que los datos

\footnotetext{
${ }^{7}$ Algunas de las críticas se pueden ver en Larson y Wilford (1979, pp. 582-583), Hicks y Streeten (1979, pp. 576577$)$, Todaro (1988, pp. 139-140), Woods (1993, p. 216) y Murray (1993, pp. 41, 47). Morris y McAlpin (1982, pp. 19-20) contestaron a parte de las mismas señalando que el cálculo del PNB también incluía importantes arbitrariedades, que una redistribución de las ponderaciones entre los tres indicadores no cambiaba apenas los resultados, mientras que el tamaño del rango de cada uno de los indicadores ya corregía la arbitrariedad de la ponderación sobre el supuesto implícito de que a mayor dispersión del indicador mayor peso tendría sobre el IFCV.

${ }^{*}$ El IFCV da una ponderación de dos tercios a las variables de salud y ciertamente la esperanza de vida recoge en parte los resultados de la mortalidad infantil, pero precisamente en situaciones de baja renta se ha encontrado una asociación altamente positiva entre la mejora de la esperanza de vida y el aumento inicial del PIB per capita, que, a partir de un determinado umbral, se interrumpe, lo que vale tanto para la industrialización europea como para la experiencia de los países en vías de desarrollo tras la Segunda Guerra Mundial (Livi-Bacci, 1990, pp. 117-119, 158). En tal sentido, se ha dicho que la esperanza de vida es un indicador «compuesto ponderado de progreso» que tiene la ventaja «de capturar
} 
para su construcción son de mayor fiabilidad que los que usa la contabilidad nacional, al proceder de fuentes homogéneas, y permiten no sólo la comparación internacional, sino que su nivel de desagregación los hace susceptibles de descender a las diferencias regionales, rural-urbanas, étnicas y de género, diferencias de las que ya se ocupó Morris (1979) en su trabajo incial y que desarrolló más tarde en relación con el caso de la India ${ }^{9}$. Las posibilidades de las fuentes se reflejan también en la metodología empleada, que permite incorporar, respectivamente, el tiempo y nuevas variables físicas al índice, tales como el ingreso de calorías per capita o las estaturas medias.

En la construcción del IFCV simplemente se valora, en una escala de 0 a 100 , cada uno de los tres indicadores, donde 0 representa el peor nivel y 100 el mejor para la esperanza de vida y también (para evitar la

el impacto sobre los individuos, no sólo de los factores no mercantiles, sino también de los impuestos, las transferencias y los servicios sociales sobre el ingreso neto, sin todas las dificultades crecientes de las medidas del ingreso per capita» (Hicks y Streeten, 1979, pp. 578-579); y el propio Sen (1988, p. 13) ha afirmado que aunque la esperanza de vida es «una medida muy limitada de lo que se ha llamado "la calidad de vida", las fuerzas que llevan a la mortalidad, como la morbilidad, la mala salud, el hambre, etc., también tienden a hacer las condiciones de vida de la gente más dolorosas, precarias y frustrantes, por lo que la esperanza de vida debería servir, en cierta medida, como indicador para otras variables de importancia». Un intento de utilizar la esperanza de vida como indicador de desarrollo en comparación con el IFCV se puede ver en Silber (1983). En un trabajo clásico Usher (1973, pp. 216-226) propuso utilizar los cambios en la esperanza de vida como indicador del crecimiento económico para los casos de Canadá (1926-1968), Ceylán (1946-1963), Chile (1931-1965), Francia (1911-1964), Japón (1930-1960) y Taiwán (1952-1966). Finalmente, señalar que Adelman y Morris (1965, p. 96) demostraron que la alfabetización se encontraba dentro del grupo de componentes con mayor influencia sobre las variaciones internacionales del PIB per capita, y que en el informe del PNUD (1990, p. 10) se dice que «aprender a leer y escribir es el primer paso de una persona hacia el aprendizaje y la adquisición de conocimientos, de manera que las cifras de alfabetismo son esenciales en cualquier medición del desarrollo humano».

"Morris y McAlpin (1982, pp. 54-78). De hecho, diversos trabajos de carácter histórico han utilizado los tres componentes del IFCV por separado con tales propósitos y en relación también con las desigualdades sociales. Woods, Watterson y Woodward (1988, pp. 353-358) y Williamson (1991, pp. 126-129) han estudiado las diferencias regionales, rural-urbanas y de clase, en Inglaterra y Gales entre 1860 y 1910 , a partir de las tasas de mortalidad infantil. Con la tasa de mortalidad infantil de los menores de cinco años y la esperanza de vida, Haines (1985) ha logrado medir de manera plausible la desigualdad social en Inglaterra y Gales y Estados Unidos a principios del siglo Xx. Gómez Redondo (1992, pp. 44-62, 204-219) ha comprobado la evolución de las disparidades territoriales y de género en la tasa de mortalidad infantil española en el siglo xx. Y Núñez (1992, pp. 89-199) y Zamagni (1993, pp. 200-217) han presentado una información muy completa sobre las diferencias regionales y (la primera autora también) de género en la alfabetización de España e Italia, un indicador que ya había servido a Good (1981, p. 144) para medir el grado de integración económica del imperio austro-húngaro. 
incoherencia de Morris) la alfabetización [1], mientras que 100 representa el peor nivel y 0 el mejor para la mortalidad infantil [2]:

$$
\frac{\mathrm{x}_{\mathrm{x}}-\mathrm{x}_{\text {mín }}}{\mathrm{x}_{\text {máx }}-\mathrm{x}_{\text {min }}} \cdot 100
$$

siendo $\mathrm{x}_{\min }$ el valor más bajo (en este caso el de peor nivel); $\mathrm{x}_{\mathrm{x}}$ el valor a convertir a la escala 0 a 100 , y $x_{\text {máx }}$ el valor más alto (en este caso el de mejor nivel);

$$
100-\frac{x_{x}-x_{\min }}{x_{\text {máx }}-x_{\min }} \cdot 100
$$

teniendo en cuenta que aquí el valor más bajo $x_{\min }$ corresponde al mejor nivel, mientras que el más alto $x_{\text {máx }}$ al peor. Una vez que las escalas son comparables, el IFCV se calcula como la media aritmética de las tres. Como es obvio, la identificación de los valores máximo y mínimo de la escala se puede hacer para la población de un solo año (los que denominaremos IFCV estático) o considerando los valóres máximo y mínimo de una población de varias fechas testigo (IFCV dinámico). En el primer caso, los resultados obtenidos no admiten comparación con los de otra fecha, sino únicamente entre sí; en el segundo, los resultados obtenidos admiten tanto la comparación interna como a lo largo del tiempo.

Tras las sugerencias de Morris de aplicar el IFCV al análisis histórico ${ }^{10}$, se debe a Federico y Toniolo (1991) la primera elaboración en el contexto de la industrialización europea, con el propósito de analizar el atraso de Italia en una perspectiva temporal (1870-1910) y comparativa (Inglaterra y Gales, Francia y Bélgica), que tuviera en cuenta también las desigualdades regionales del país. Sus conclusiones apuntan, en primer lugar, a que Italia como país estaba muy lejos del nivel de vida de las economías adelantadas de Europa, aunque su IFCV (dinámico) se acercó más deprisa al de sus competidores que lo que lo hizo su PIB per capita (el ranking del IFCV coincidía en todo caso con el del PIB per capita). Y, en segundo lugar,

10 Véase su reconstrucción para los Estados Unidos entre 1900 y 1974 (Morris, 1979, p. 79), la India entre 1901 y 1971 y Sri Lanka entre 1921 y 1971 (Morris y McAlpin, 1982, pp. 84-85). 
las tres grandes regiones italianas presentaban enormes diferencias entre sí: hacia 1910, el noroeste estaba muy cerca del IFCV de los países adelantados, mientras que Italia como conjunto (y su gran región norte y este) estaba en una posición intermedia mucho más alejada, y el sur se encontraba más cerca probablemente de Rusia que de la media de Italia. A la vista de estas conclusiones, Federico y Toniolo establecieron que era «muy difícil discutir el atraso italiano en términos agregados».

En el Cuadro 1 hemos recogido los datos que permiten reconstruir el IFCV estático para una muestra más amplia de países europeos en torno a las fechas de 1900-1914. Su comparación con el ranking Borda ${ }^{11}$ del producto per capita resultante de las estimaciones de Bairoch, Crafts y Maddison es bastante expresiva. En primer lugar, los países colistas en el ranking Borda del producto per capita presentan idénticas posiciones en el del IFCV (España, Hungría y Rusia) o muy similares (Italia), y, al contrario, los países mejor situados en el ranking del producto per capita presentan IFCV elevados (Reino Unido, Holanda y, en menor medida, Francia). Aunque la correlación estadística entre ambos indicadores es elevada ${ }^{12}$, en varios casos hay una relación asimétrica entre producto per capita e IFCV. Ello sugiere que se pueden conseguir mejoras tan importantes en la calidad de vida antes de lograr un incremento del producto per capita, que el país en cuestión se puede situar como líder en términos amplios de bienestar mientras todavía permanece en una posición intermedia en producto per capita (caso de Suecia); y, a la inversa, un nivel elevado de producto per capita puede ser a costa de una lenta mejora en la calidad de vida (caso de Austria y, en menor medida, de Alemania). ¿Qué decir de los casos de España e Italia? En el país transalpino ya se vio cómo la media nacional del producto per capita y del IFCV era muy poco significativa, por cuanto las diferencias entre las regiones ricas y las pobres resultaban muy acusadas: de hecho, de los datos de Federico

$"$ Dicho ranking es el resultante de asignar una ordenación de 1 a $n$ a la suma de los rankings respectivos de cada una de las tres estimaciones, que, a su vez, se ordenan sobre el supuesto de que 1 corresponde siempre al valor más alto y $n$ al más bajo. El invento de Borda se publicó en 1781 en las Memorias de la Real Academia de Ciencias de Paris (Rowley, 1987) y uno de los primeros en utilizarlo en el ámbito de la economía del desarrollo fue Sen (1981, pp. 310-311). Más tarde, Crafts (1993) y Dasgupta y Weale (1992) han hecho uso de esta herramienta para construir sendos indicadores alternativos de bienestar.

12 El coeficiente de correlación de rangos de Spearman para el ranking de IFCV y el de producto per capita es de 0,77 , pero hay que tener en cuenta que uno de los componentes de este último (la estimación de Crafts) toma entre otras variables para deducir el PIB per capita la tasa de mortalidad infantil. 


\section{CUADRO 1}

IFCV en Europa c. 1900-1914

\begin{tabular}{|c|c|c|c|c|c|}
\hline Paises & $\begin{array}{c}\text { Esperanza } \\
\text { de vida }\end{array}$ & $\begin{array}{c}\text { Mortalidad } \\
\text { infantil }\end{array}$ & $\begin{array}{l}\text { Alfabe- } \\
\text { tización }\end{array}$ & $I F C V$ & $\begin{array}{c}\text { Ranking prod } \\
\text { per capita }\end{array}$ \\
\hline & $*$ & $1906-1915$ & $1900-1901$ & & c. $1913^{* *}$ \\
\hline Suecia (a) ................. & 55,7 & 75 & 81 & 91,04 & 6 \\
\hline Reinc Unido (b)...... & 53,5 & 113 & 97 & 88,78 & 1 \\
\hline Holanda (c) ........... & 52,2 & 107 & $81(c)$ & 80,08 & 2 \\
\hline Francia (d) & 50,4 & 127 & 82 & 74,28 & 5 \\
\hline Alemania (e) ............ & 49,0 & 167 & 99 & 73,28 & 3 \\
\hline Checoslovaquia (f). & 40,3 & 203 & 96 & 52,64 & 7 \\
\hline Italia $(\mathrm{g}) \ldots \ldots \ldots \ldots \ldots$ & 46,9 & 146 & 52 & 50,81 & 8 \\
\hline Austria (h) ............. & 40,1 & 190 & 77 & 45,32 & 4 \\
\hline España (i) ............... & 41,7 & 156 & 45 & 38,02 & 9 \\
\hline Hungría (j) ............. & 37,5 & 209 & 59 & 29,11 & 10 \\
\hline Rusia $(\mathrm{k}) \ldots \ldots \ldots \ldots \ldots$ & 32,4 & $254(k)$ & 32 & 0,00 & 11 \\
\hline
\end{tabular}

* Esperanza de vida: (a): 1901-1910; (b): Inglaterra y Gales, 1910-1911; (c): 1900-1909, corresponde a Bélgica; (d): 1908-1913; (e): 1910-1911; (f): 1899-1902; (g): 1910-1912; (h): 1900-1901; (i): 1910; (j): 1900-1901; (k): 1897, y para mortalidad infantil 1910-1914.

** Sobre los cálculos de Bairoch para 1913, Crafts (con datos de Davis para los países desgajados del antiguo imperio austro-húngaro y nueva estimación de Federico y Toniolo para Italia) para 1910 y Maddison para 1913.

Fuente: Vallin (1989, p. 37) para esperanza de vida, completado en algunos países con Livi-Bacci (1990, p. 115; 1993, p. 140); Gómez Redondo (1993, p. 38) para mortalidad infantil, completado en algúnos países con Toniolo (1988, p. 59); Cipolla (1970, pp. 17, 151-154, 157) para alfabetización, completado con Mirónov (1993, p. 299) y Núñez (1993, p. 372). Los datos para el ranking de PIB per capita en Bairoch (1981, p. 10), Crafts (1984, p. 440); Good (1991, pp. 223-224, 228, 230), Federico y Toniolo (1991, p. 199) y Maddison (1991, pp. 12, 26).

y Toniolo (1991) se deduce que hacia 1910 la zona noroeste de Italia tenía un PIB per capita equivalente al 92 por 100 del de Francia, mientras que la media de Italia se quedaba en el 68 por 100 y la del sur del país en el 51 por 100, muy cerca del 45 por 100 que suponía el PIB per capita de Rusia como porcentaje del de Francia. Con el IFCV, tomando las mismas referencias, el norte tenía un 84 por 100 , Italia un 74 por 100 y el sur del país un 59 por 100 del IFCV francés. En el caso de España, un pequeño ejercicio muestra las nítidas semejanzas que para la misma época había con la otra nación latina. 
En el Cuadro 2 se presenta el IFCV estático de aquellas regiones cuyos valores para los tres indicadores se encuentran dentro del rango de los países seleccionados del Cuadro $1^{13}$. Esto implica dejar fuera a algunas (en concreto Baleares, Canarias y Murcia, cuyas cifras de alfabetización resultan ligeramente inferiores a las de Rusia, pese a que en las otras variables, las dos primeras regiones presentan valores sensiblemente mejores que los rusos). Los resultados obtenidos permiten deducir tres conclusiones: 1) que las tres regiones norteñas más próximas geográficamente a Francia (País Vasco, Navarra y Cantabria) tenían en torno al 85 por 100 del IFCV francés, una magnitud muy similar a la del norte de Italia ( 84 por 100) respecto al mismo país, y a la que se acercaban Asturias y Cataluña (73 y 70 por 100 respectivamente); 2) que el IFCV de España estaba mucho más alejado que el de Italia del referente francés ( 51 contra 68 por 100), debido al enorme lastre de las regiones interiores y del sur (salvando la isla madrileña), y 3) que la diferencia española se marca también en el carácter más acusado del atraso de nuestro Mezzogiomo, las regiones de Castilla-La Mancha, Andalucía y Extremadura, que se situaban entre el 35 y el 30 por 100 del IFCV francés, mucho más lejos que el sur de Italia ( 59 por 100 ), y en una posición inmediatamente por debajo del IFCV de Hungría con el único suelo del imperio ruso. Esta consideración de las fuertes disparidades regionales españolas indica que el IFCV, en la medida que se construya para períodos históricos amplios, puede servir de complemento de otros cálculos del producto per capita a nivel regional ${ }^{14}$.

13 Para ello nos hemos valido de la alfabetización adulta en 1900, calculada como una ratio en \% entre el número total de personas alfabetizadas y la población mayor de 10 años (Censo de la población de España... de 1900. Madrid, 1903, vol. I, pp. 477-479; y 1907, vol. III, pp. X.XI), de los datos de esperanza de vida de 1910 (Dopico y Reher, 1998, p. 43) y de las tasas de mortalidad infantil de 1911-1915 en promedio artimético de las provincias correspondientes en caso de regiones pluriprovinciales (Gómez Redondo, 1992, p. 376). Los datos espanoles de alfabetización y de mortalidad infantil pueden estar algo infravalorados, pero, dada la consideración en el índice como valor máximo del más elevado para la alfabetización y del más bajo para la mortalidad infantil (con el valor mínimo al contrario), no creemos que la performance de España y de las regiones con peor IFCV en esa muestra se llegara a situar por debajo de la de Hungría; por tanto, la posición en el ranking de España quedaría igual y la de las regiones con menor IFCV muy similar si se utilizara la alfabetización tomando la referencia a los mayores de 6.8 años y se pudiera corregir la mortalidad infantil.

${ }_{14}$ Véase especialistas de signos teóricos tan opuestos como Beckerman (1993, p. 95) y Desai (1993, p. 28). De hecho, hemos realizado un ejercicio tentativo para relacionar el ranking de renta per capita a nivel provincial de 1964 con el del IFCV, construido con datos de esperanza de vida al nacer —en realidad, un promedio de la esperanza de vida masculina y femenina para 1961-1965-, la tasa de mortalidad infantil sin corregir para 


\section{CUADRO 2}

IFCV en Europa y las regiones españolas, c.1900-1914

\begin{tabular}{|c|c|c|c|c|c|c|}
\hline Países & $\begin{array}{c}\text { Esperanza } \\
\text { de vida }\end{array}$ & $\begin{array}{c}\text { Mortalidad } \\
\text { infantil } \\
\end{array}$ & $\begin{array}{l}\text { Alfabe- } \\
\text { tización }\end{array}$ & $I F C V$ & & \\
\hline 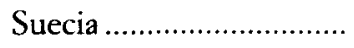 & 55,7 & 75 & 81 & 91,04 & & \\
\hline Reino Unido........................ & 53,5 & 113 & 97 & 88,78 & & \\
\hline 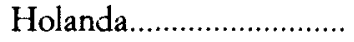 & 52,2 & 107 & 81 & 80,08 & & \\
\hline 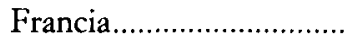 & 50,4 & 127 & 82 & 74,28 & $=$ & 100 \\
\hline Alemania & 49,0 & 167 & 99 & 73,28 & & \\
\hline Checoslovaquia .................. & 40,3 & 203 & 96 & 52,64 & & \\
\hline 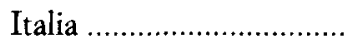 & 46,9 & 146 & 52 & 50,81 & & 68 \\
\hline 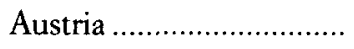 & 40,1 & 190 & 77 & 45,32 & & \\
\hline España & 41,7 & 156 & 45 & 38,02 & & 51 \\
\hline País Vasco ....................... & 46,5 & 112 & 67 & 63,98 & & 86 \\
\hline Navarra ......................... & 46,7 & 114 & 67 & 63,96 & & 86 \\
\hline Cantabria ......................... & $*$ & 128 & 76 & 62,24 & & 84 \\
\hline Asturias............................ & 45,0 & 120 & 55 & 54,54 & & 73 \\
\hline 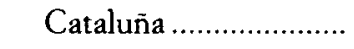 & 43,9 & 115 & 51 & 51,76 & & 70 \\
\hline 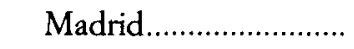 & $*$ & 167 & 76 & 47,63 & & 64 \\
\hline Galicia ............................ & 46,4 & 121 & 35 & 46,41 & & 62 \\
\hline 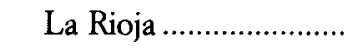 & $*$ & 159 & 64 & 45,73 & & 62 \\
\hline Castilla y León.............. & $*$ & 168 & 64 & 41,68 & & 56 \\
\hline Valencia .......................... & 43,7 & 121 & 32 & 40,93 & & 55 \\
\hline 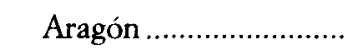 & 40,0 & 151 & 42 & 35,16 & & 47 \\
\hline Castilla-La Mancha .... & $*$ & 178 & 37 & 26,00 & & 35 \\
\hline 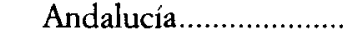 & 39,2 & 175 & 33 & 25,00 & & 34 \\
\hline Extremadura........................ & 39,6 & 195 & 34 & 22,37 & & 30 \\
\hline 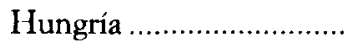 & 37,5 & 209 & 59 & 29,11 & & \\
\hline 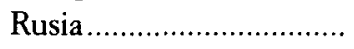 & 32,4 & 254 & 32 & 0,00 & & \\
\hline
\end{tabular}

* No se incluyen por no haber sido publicados.

FUENTE: Cuadro 1 y nota 13. El IFCV correspondiente a España debería variar ligeramente respecto al del Cuadro 1, ya que la tasa de mortalidad infantil para 1911-1915 es de 152,4 por 1.000 , pero ello resulta irrelevante en la posición relativa dentro del ranking europeo de IFCV.

el mismo periodo y la tasa bruta de alfabetización de 1960. El coeficiente de correlación de rangos de Spearman arroja un valor positivo elocuente $(0,69)$. Las fuentes se encuentran en Banco Bilbao (1978, p. 11), Devolder (1985, p. 23), Gómez Redondo (1992, p. 381) y Censo de la población... de 1960, Madrid, vol. III. 
Para terminar este apartado hemos de señalar que las variables que componen el IFCV para las provincias y regiones españolas muestran un grado de correlación, en general, menor que las que se encuentran en el trabajo de Morris y que Larson y Wilford (1979) señalaron como elemento de debilidad del mismo o de superfluidad de su índice. Esta menor correlación se presenta sobre todo para las variables demográficas confrontadas con la alfabetización en cualquiera de los años y las escalas elegidas, mientras que la esperanza de vida y la mortalidad infantil, aun estando menos correlacionadas que las de Morris, presentan unos niveles similares a los de otros países durante la misma época y tienen una evolución convergente (Cuadro 3) ${ }^{15}$. Pero el hecho, mostrado por Murray (1993), de que el declive en la mortalidad infantil pueda enmascarar en la esperanza de vida al nacer un total estancamiento e incluso un empeoramiento en las condiciones de salud y mortalidad de los adultos (que podría darse quizá en nuestro caso en 1860) aconseja incluir ambos indicadores en el IFCV, y quizá por ello lo hizo Morris en su día.

\section{EL IFCV EN ESPAÑA 1860-1930: ANÁLISIS DE LOS RESULTADOS}

Antes de cualquier otra observación es necesario remarcar que el grado de dispersión provincial de la calidad física de vida aumentó entre 1860 y 1900, como demuestra el coeficiente de variación del IFCV estático, que pasó de 0,29 a 0,47 entre ambas fechas, para reducirse luego al 0,46 en 1910 y al 0,39 en 1930. A nivel regional, el coeficiente de variación del IFCV estático entre 1900 y 1930 se mantuvo estancado en el 0,46. Ello introduce un elemento de escepticismo acerca de la validez de los datos iniciales de renta regional de Álvarez Llano (1986), reconvertidos en términos per capita relativos por el procedimiento de Carreras (1990), cuyo coeficiente de variación fue descendiendo desde el 0,54 de 1860 al 0,37 de 1900 y 0,33 de 1930 . Por tanto, cualquier extrapolación de los coeficientes de variación del IFCV como alternativa para medir la dispersión de la renta regional resulta sumamente arriesgada, puesto que el ranking de IFCV estático a nivel regional y el de producto por habitante presentan una correlación de rangos de Spearman positiva tan sólo del

15 Woods $(1993$, pp. 198, 202) ha encontrado un valor del coeficiente de correlación de $-0,64$ para Inglaterra y Gales en la década de 1860 y de $-0,94$ para Suecia en la siguiente. 
CUADRO 3

Matriz de correlaciones para las variables del IFCV estático a nivel provincial, $1860-1930$

\begin{tabular}{|c|c|c|c|c|}
\hline & Variables & Esperanza de vida & Mortalidad infantil & Alfabetización \\
\hline \multirow{3}{*}{1860} & esperanza de vida & 1,00 & & \\
\hline & mortalidad infantil & $-0,57$ & 1,00 & \\
\hline & alfabetización & $-0,29$ & 0,12 & 1,00 \\
\hline \multirow{3}{*}{1900} & esperanza de vida & 1,00 & & \\
\hline & mortalidad infantil & $-0,84$ & 1,00 & \\
\hline & alfabetización & $-0,02$ & $-0,04$ & 1,00 \\
\hline \multirow{3}{*}{1910} & esperanza de vida & 1,00 & & \\
\hline & mortalidad infantil & $-0,89$ & 1,00 & \\
\hline & alfabetización & 0,07 & $-0,17$ & 1,00 \\
\hline \multirow{3}{*}{1930} & esperanza de vida & 1,00 & & \\
\hline & mortalidad infantil & $-0,88$ & 1,00 & \\
\hline & alfabetización & 0,11 & $-0,01$ & 1,00 \\
\hline
\end{tabular}

Fuente: Anexo 1.

0,41 para 1900 , y del 0,56 para 1930 , si bien no es despreciable la tendencia convergente que se observa.

En segundo lugar, los resultados del IFCV estático a nivel provincial (mapas 1 al 3) muestran la mala situación hacia 1860 de las provincias del interior peninsular, incluido Madrid (y la Cataluña interior), y del sur, todas ellas por debajo del nivel medio español; en el extremo opuesto, destacan las provincias norteñas, Baleares, algo más retrasada la Cataluña costera y un grupo mixto de excepciones (Sevilla, Huelva, Salamanca, Palencia o Soria) que dejan de serlo por su cercanía a la media nacional. Con el paso del tiempo esta dualidad norte-interior tiende a homogeneizarse, de manera que hacia 1900 quedan muy por debajo de la media nacional todas las provincias andaluzas (salvo la excepción de Huelva) y también por debajo están toda Castilla-La Mancha, Extremadura y Murcia; en el extremo superior, se encuentra la Cornisa cantábrica, Navarra, Cataluña y Baleares, $y$, superando ligeramente la media nacional, Madrid y las provincias de la Comunidad Valenciana, mientras que en torno a la media - como ocurre en Italia con su zona central - gravitan la mayoría 


\section{MAPA 1: IFCV estático, 1860.}

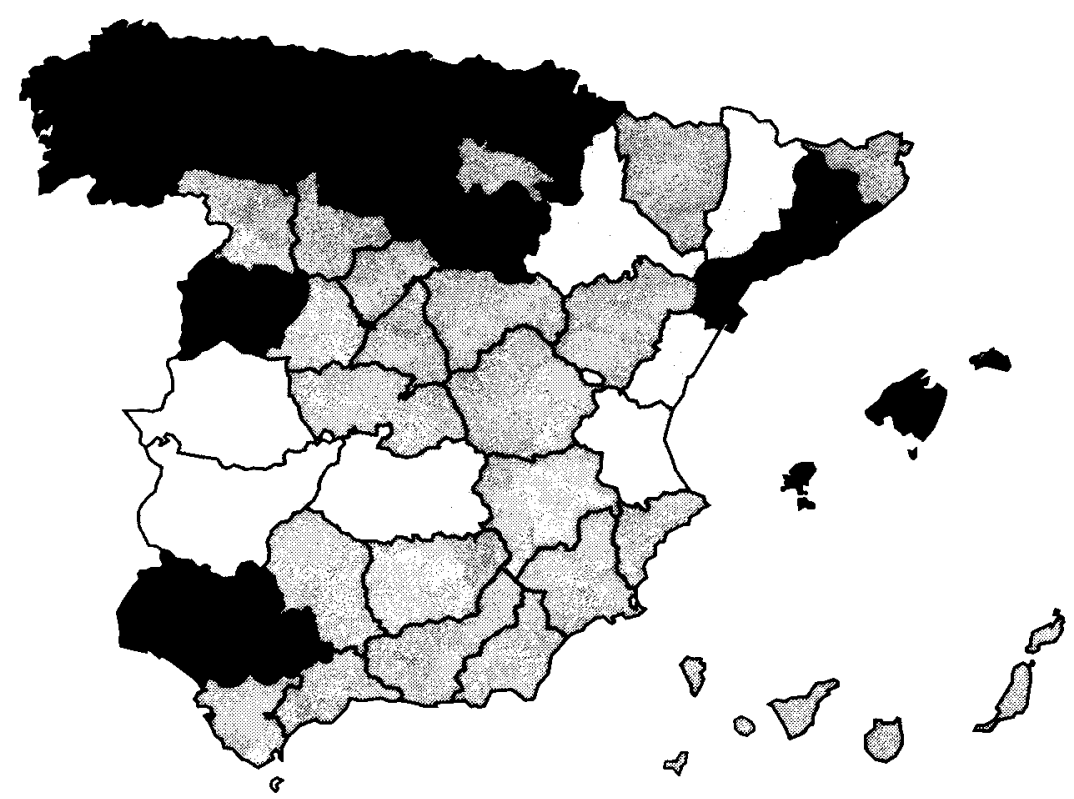

\begin{tabular}{|l|l|}
\hline \multicolumn{2}{|c|}{ Variación sobre la media } \\
más del $50 \%$ & $\square$ de 0 a $-25 \%$ \\
entre 25 y $50 \%$ & $\square$ de -50 a $-25 \%$ \\
entre 0 y $25 \%$ & $\square$ inferior a $-50 \%$ \\
\hline
\end{tabular}

de las provincias de Castilla y León, La Rioja y Aragón (con la excepción de Zaragoza). En 1930, estas diferencias se estabilizan, con algunos cambios internos significativos dentro de la jerarquía de cada grupo: en el extremo superior las provincias catalanas se sitúan a la cabeza del ranking, mientras Cantabria desciende de posición por debajo de las vascas; el grupo extremeño, andaluz y castellano-manchego en el extremo inferior, al que se incorporaba Ávila, se ve engrosado ahora por otras provincias castellano-leonesas como Zamora y Palencia, además de Canarias.

En tercer lugar, el IFCV dinámico en España entre 1860 y 1930 mejoró tanto en términos agregados como a nivel provincial. Para analizar el ritmo de dicha mejora se ha utilizado la tasa de reducción de la disparidad (TRD) 


\section{MAPA 2: IFCV estático, 1900.}

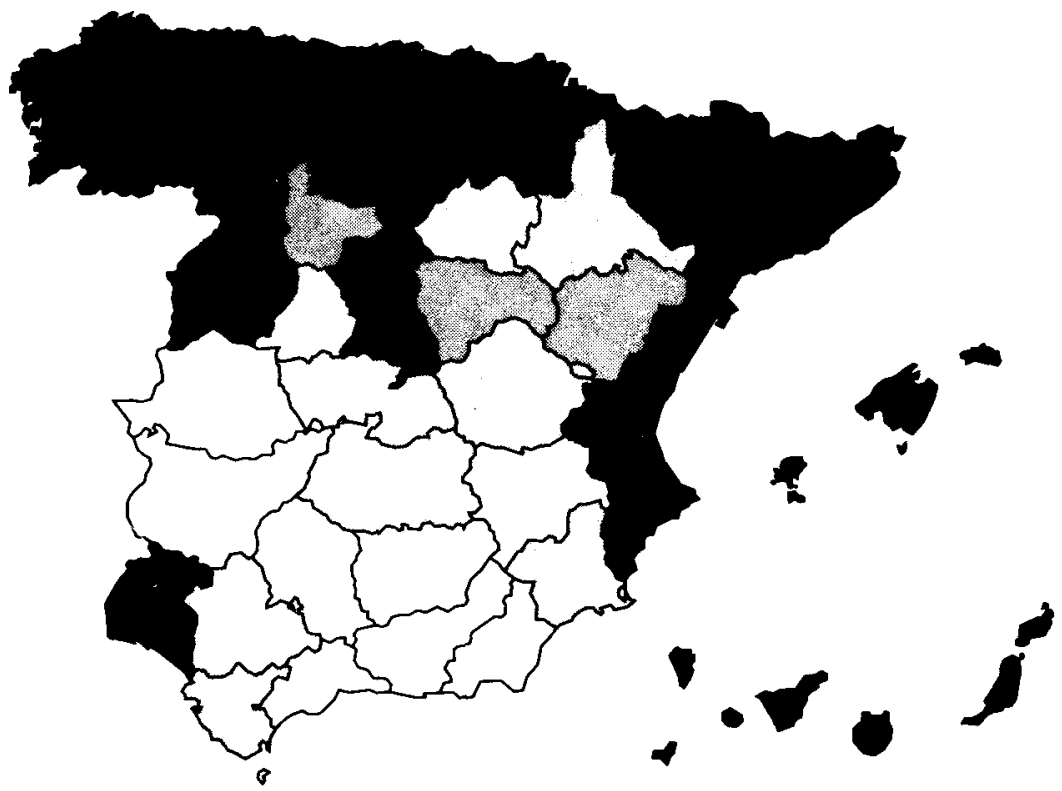

\begin{tabular}{|l|l|}
\hline \multicolumn{3}{|c|}{ Variación sobre la media } \\
más del $50 \%$ & $\square$ de 0 a $-25 \%$ \\
entre 25 y $50 \%$ & $\square$ de -50 a $-25 \%$ \\
entre 0 y $25 \%$ & $\square$ inferior a $-50 \%$ \\
\hline
\end{tabular}

de Morris (1979) y Morris y McAlpin (1982): en realidad, una tasa de crecimiento acumulativo anual del IFCV dinámico, que se interpreta en el mismo sentido que la tasa de crecimiento del producto o el ingreso per capita. El propósito de estos autores era comparar la evolución del IFCV dinámico y del producto per capita, lo que hemos hecho para España con los siguientes resultados. La TRD para el período 1860-1930 creció más rápido $(1,5)$ que el $\mathrm{PIB}$ real per capita $(0,9)$ en el periodo similar (1860-1929); si se comparan períodos intermedios esa diferencia se mantiene antes de la I Guerra Mundial (1,4 la TRD para 1860-1910, frente al 0,9 del PIB real per capita entre $1860-1913)^{16}$, tras la cual parece que

${ }^{16}$ Otro paralelismo más con Italia, donde la TDR aumentó al 2,1 mientras que el 

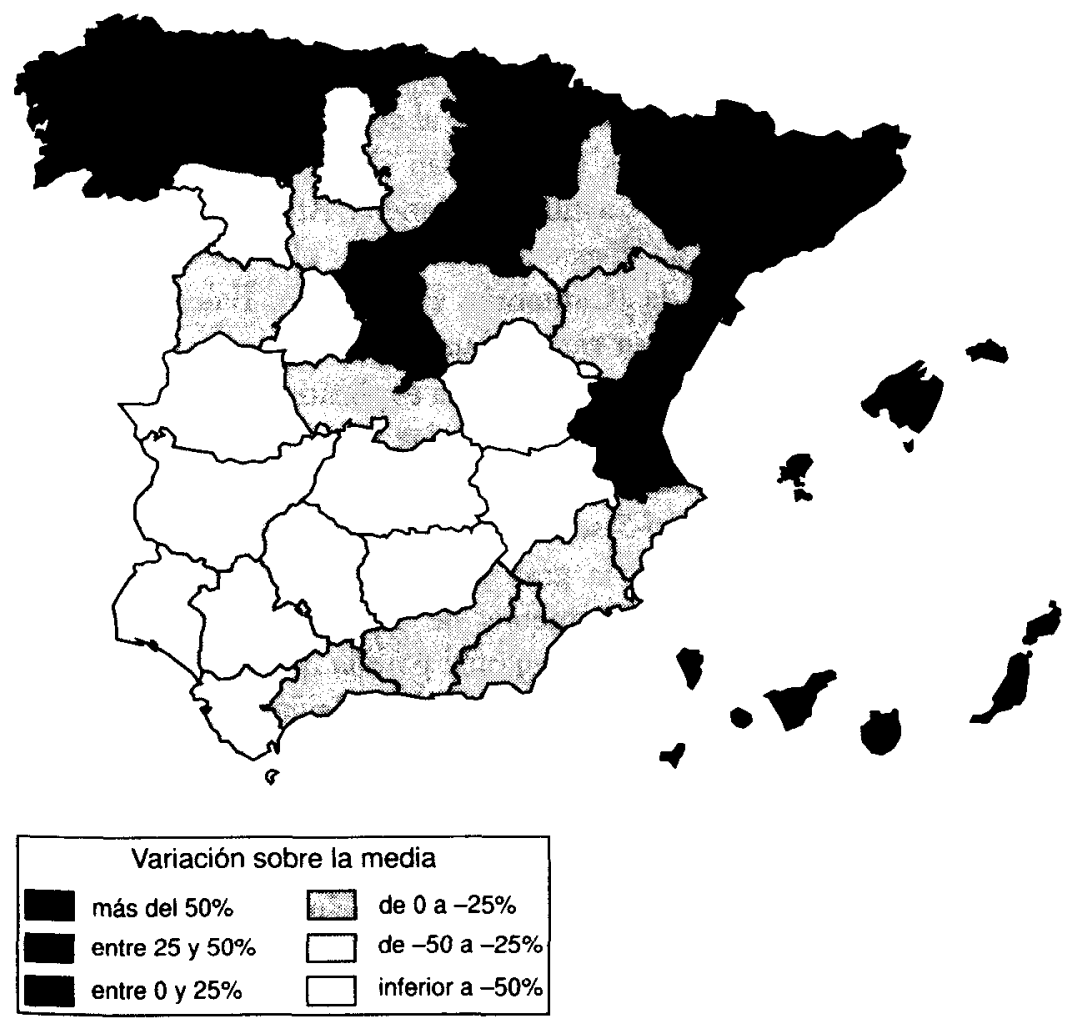

las variables crecieron al mismo ritmo (1,4 la TRD para 1910-1930 y 1,4 el PIB real per capita para 1913-1929) ${ }^{17}$. Al igual que en Italia, la mejora en el índice podría reflejar que la relación entre el PIB per capita y el IFCV dibuja una función creciente a tasa decreciente: si suponemos como dada la existencia de una tasa de crecimiento del PIB per capita a nivel provincial, las provincias que partian en 1860 de niveles de IFCV más bajos mejoraron hasta 1930 más rápido que las que tenían niveles de IFCV

producto per capita lo hizo al 1,5 para el período 1870-1910 (Federico y Toniolo, 1991, pp. 199, 201).

17 Los datos de la TRD en Cuadro 4. Los del PIB per capita para 1860-1930 en Prados (1993, pp. 289, 296), Bardini, Carreras y Lains (1995, p. 142) y Simpson (1997a, pp. 348-349). 
más altos (Anexo 2) y, de hecho, la correlación entre el ranking de IFCV dinámico en 1860 y la TRD para 1860-1930 resulta altamente negativa $(-0,90)$ (en Italia la TRD de la región del sur creció al 2,6 mientras que la del noroeste lo hizo al 1,4 entre 1870 y 1910). Si se utilizan los datos a nivel regional, dicha hipótesis se confirma al comparar el IFCV dinámico entre 1900 y 1930: las regiones que partían de peores posiciones (Extremadura, Andalucía, Murcia, Castilla-La Mancha, Aragón y Valencia) fueron las que más redujeron su disparidad, mientras que las que tenían un IFCV más elevado de partida (Cantabria, País Vasco, Baleares, Navarra y Asturias) lo mejoraron a menor ritmo (Cuadro 4).

En contraste con la previsible irrelevancia explicativa de la urbanización en los resultados del IFCV ${ }^{18}$, el peso que las estructuras agrarias tenían todavía en la España de principios de siglo tuvo que condicionar los resultados del IFCV. En el Cuadro 5 se comparan las estimaciones disponibles sobre el peso de la pequeña y la gran propiedad en España, expresado como el porcentaje de fincas de menos de 10 y de más de 250 Has., respectivamente, sobre la superficie regional para la que existen datos, con el IFCV estático de 1930 también a nivel regional. Asignando al País Vasco, Cantabria y Asturias el puesto 2 del ranking en IFCV, el coeficiente de correlación de rangos de Spearman entre dicho ranking y el de la pequeña propiedad arroja un valor de $0,73 \mathrm{y}$, si se toma como referencia la gran propiedad, de $-0,87$. Es obvio, por tanto, que el predominio del minifundio está generalmente asociado con los valores más elevados de IFCV, con la notable excepción de Galicia, donde quizá la fragmentación de las explotaciones fue demasiado lejos y que la España que presenta los IFCV más bajos coincide con el área de mayor peso de la gran propiedad, las regiones de Andalucía, Castilla-La Mancha y Extremadura. Creemos que

${ }^{18}$ Aunque la urbanización es uno de los cambios estructurales que acompañan y refuerzan el crecimiento del PIB per capita, también está asociada con niveles de mortalidad infantil y de alfabetización más elevados, de manera que, a la vista de cómo está construido el IFCV, no parece que se pueda hallar ninguna correlación significativa entre el aumento del mismo y el de la urbanización. A partir de los coeficientes de urbanización a nivel provincial elaborados por Luna (1988, pp. 62-65) para 1860 (ayuntamientos mayores de 5.000 habitantes), 1887, 1900, 1910 y 1930 (ayuntamientos cuyo casco urbano supera los 5.000 habitantes) se han realizado diversas pruebas, pero los resultados son muy poco concluyentes. En concreto, existe una correlación negativa entre los rankings de IFCV dinámico y los de urbanización, pero resulta muy débil: $-0,23$ para $1860,-0,21$ para 1910 y $-0,18$ para 1930. El ranking de la TRD para 1860-1930 y el del aumento de la urbanización para 1887-1930 (con los datos homogéneos sobre casco urbano) tienen un valor del coeficiente de $-0,10$, lo que avala la hipótesis de que el IFCV y el proceso general de urbanización en España entre 1860 y 1930 no tuvieron apenas correlación. 


\section{CUADRO 4}

IFCV dinámico en España a nivel regional, 1900-1930 $y$ tasas de reducción de la disparidad

\begin{tabular}{|c|c|c|c|c|c|c|}
\hline \multirow{2}{*}{$\frac{\text { Regiones }}{\text { Cantabria .................... }}$} & \multicolumn{2}{|c|}{ IFCV 1900} & \multicolumn{2}{|c|}{ IFCV 1930} & \multicolumn{2}{|c|}{$T R D$} \\
\hline & 49,43 & (1) & 85,76 & (3) & 1,85 & (17) \\
\hline País Vasco.. & 44,95 & (2) & 90,02 & (1) & 2,34 & (13) \\
\hline Baleares ...... & 44,41 & (3) & 85,37 & (4) & 2,20 & $(15 / 16)$ \\
\hline Navarra.. & 43,24 & (4) & 83,30 & (6) & 2,21 & (14) \\
\hline Asturias ..... & 42,54 & (5) & 83,54 & (5) & 2,20 & $(15 / 16)$ \\
\hline Cataluña . & 36,67 & (6) & 87,51 & $(2)$ & 2,94 & (9) \\
\hline Galicia............................ & 34,92 & (7) & 71,47 & (9) & 2,42 & (12) \\
\hline Castilla y León... & 29,79 & $(8)$ & 66,96 & $(12)$ & 2,74 & (11) \\
\hline La Rioja ............. & 28,46 & (9) & 72,36 & (7) & 3,16 & $(8)$ \\
\hline Madrid ............. & 28,22 & $(10)$ & 74,55 & $(8)$ & 3,29 & (7) \\
\hline Canarias..... & 25,24 & (11) & 57,16 & (15) & 2,76 & $(10)$ \\
\hline Valencia. & 24,83 & (12) & 69,17 & (11) & 3,47 & (6) \\
\hline Aragón ................... & 19,65 & (13) & 70,00 & (10) & 4,32 & (5) \\
\hline Castilla-La Mancha ..... & 14,20 & (14) & 56,55 & (16) & 4,71 & (4) \\
\hline Murcia ......................... & 10,05 & (15) & 60,42 & (13) & 6,16 & (3) \\
\hline Andalucía ..................... & 8,29 & (16) & 59,69 & (14) & 6,80 & (2) \\
\hline Extremadura... & 4,41 & (17) & 48,93 & (17) & 8,35 & (1) \\
\hline
\end{tabular}

FUENTE: Ver Cuadro 6; entre paréntesis rankings respectivos.

estos resultados indican que la distribución de la propiedad no es un elemeno neutral a la hora de explicar las diferencias en los niveles de vida y, por ello también, nuestros resultados empiezan a aclarar el panorama de las relaciones entre estructura de la propiedad de la tierra y obstáculos al desarrollo, que Tortella (1994) apuntó como elemento institucional común de las naciones latinas. Igualmente significativos son los resultados de la comparación del ranking del IFCV estático a nivel regional (con todas las regiones menos Canarias) con los de la productividad del trabajo y de la tierra para 1930 de Gallego (1993): los coeficientes de correlación de rangos de Spearman son del 0,78 y del 0,82 , respectivamente ${ }^{19}$.

${ }^{19} \mathrm{Al}$ comparar los rankings de IFCV estático a nivel provincial y los de la productividad del trabajo agrícola y de la tierra de 1931 (Simpson, 1994, p. 77), los resultados son del 0,36 y del 0,60 , respectivamente. Esta mayor correlación entre IFCV y productividad de la tierra, que se amplía en cuanto pasamos del nivel regional al provincial, avala nuestras 


\section{CUADRO 5}

IFCV estático en 1930 y estructura de la propiedad de la tierra

\begin{tabular}{|c|c|c|c|}
\hline Regiones & IFCV 1930 & $\begin{array}{l}\% \text { fincas }<10 \text { Has. } \\
\text { sobre superficie total * }\end{array}$ & $\begin{array}{l}\% \text { fincas }>250 \text { Has. } \\
\text { sobre superficie total * }\end{array}$ \\
\hline País Vasco......................... & $78,73\}$ & & \\
\hline 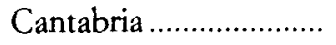 & $69,68 \mid$ & 69,2 & 4,9 (a) \\
\hline 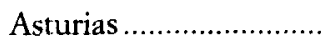 & $68,22\}$ & & \\
\hline Cataluña ................... & 77,45 & 54,4 & 5,5 \\
\hline 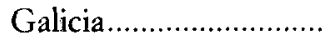 & 49,48 & 78,7 & 4,6 \\
\hline Valencia . & 44,43 & 51,4 & 14,6 \\
\hline 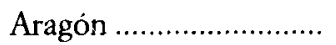 & 43,76 & 54,7 & $11,3(\mathrm{~b})$ \\
\hline Castilla y León............. & 33,63 & 66,1 & $14,4(\mathrm{c})$ \\
\hline Murcia ........................ & 29,57 & 33,4 & $20,5(d)$ \\
\hline Andalucía ................... & 26,03 & 24,4 & $44,6(\mathrm{e})$ \\
\hline Castilla-La Mancha ..... & 19,84 & 34,8 & $38,8(f)$ \\
\hline Extremadura .................... & 5,67 & 27,4 & $35,8(\mathrm{~g})$ \\
\hline
\end{tabular}

* (a): el País Vasco sin Álava, (b): incluye Rioja, (c): sin Salamanca, (d): incluye Almería, (e): sin Almería, (f): sin Cuenca ni Guadalajara, (g): incluye Salamanca.

Fuevte: Cuadro 6, Carrión (1973, p. 40) y Malefakis (1980, pp. 32, 35).

Por último, de la comparación del IFCV estático con las estimaciones de renta regional per capita en 1900 y 1930 (Cuadro 6), se pueden avanzar dos conclusiones. La primera es que existe una diferencia muy notable entre el coeficiente de correlación de rangos de Spearman que se deduce de la comparación del ranking de IFCV y producto per capita del Cuadro 1 $(0,77)$ y el que resulta de los datos del Cuadro 6 para $1900(0,41)$ y 1930

conclusiones sobre la estructura de la propiedad, puesto que precisamente la productividad de la tierra era más elevada en donde predominan las pequeñas explotaciones en propiedad (Simpson, 1997b, pp. 79-84), lo que pernitiría una ampliación de los derechos de acceso. También hemos considerado que la posibilidad de emigrar actuara en el mismo sentido, pero evidentemente ello repercutiría teóricamente sobre los resultados del IFCV en las regiones de destino, por eso la comparación del ranking provincial de la tasa bruta de emigración exterior de 1910-1920 (Sánchez Alonso, 1995, pp. 292-293) y el del IFCV estático de 1930 arroja sólo un valor de 0,25 . Además, dada la preferencia a emigrar a las ciudades, las previsibles consecuencias sobre el IFCV quedarian anuladas: en tal sentido, la comparación del ranking de IFCV estático a nivel regional de 1930 con las tasas de emigración inter-regionales (expresadas en promedios anuales por cada mil habitantes) para los periodos 1911-1920 y 1921-1930 (Mikelarena, 1993, p. 239) dan unos resultados escasamente sig. nificativos: 0,17 y 0,25 , respectivamente. 


\section{CUADRO 6}

IFCV estático y ranking de PIB per capita de España a nivel regional, 1900 y 1930

\begin{tabular}{|c|c|c|c|}
\hline IFCV 1900 & $P I B p c$ & IFCV 1930 & $P I B p c$ \\
\hline$\ldots . .74,47 \quad(1)$ & 3 & $\ldots 78,73 \quad(1)$ & 2 \\
\hline$\ldots \ldots \ldots .67,32$ & 13 & $\ldots 78,72$ & 7 \\
\hline País Vasco .............66,33 & 4 & $\ldots 77,45 \quad$ (3) & 1 \\
\hline Navarra..............64,21 & 5 & .......69,68 (4) & 10 \\
\hline Asturias.................63,81 (5) & 8 & Asturias.................68,22 (5) & 12 \\
\hline Cataluña ................54,27 (6) & 2 & 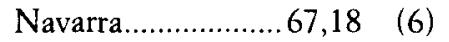 & 5 \\
\hline Galicia ................53,31 (7) & 17 & $\ldots 49,48 \quad(7)$ & 17 \\
\hline Castilla y León ....... 44,53 & 9 & $\ldots . . .46,98 \quad(8)$ & 3 \\
\hline La Rioja ................ 42,69 & 7 & La Rioja ................. 45,64 (9) & 8 \\
\hline Canarias..................41,58 (10) & 16 & $\ldots .44,43(10)$ & 4 \\
\hline Madrid....................41,06 (11) & 1 & $. .43,76(11)$ & 6 \\
\hline Valencia ..................37,19 (12) & 10 & Castilla y León .......33,63 (12) & 9 \\
\hline Aragón .....................29,08 (13) & 6 & Murcia ...................29,57 (13) & 15 \\
\hline Castilla-La Mancha 22,48 (14) & 12 & Canarias.................27,14 (14) & 16 \\
\hline Murcia …............ 15,65 (15) & 14 & $\ldots . .26,03 \quad(15)$ & $13 / 14$ \\
\hline Andalucía...............15,58 (16) & 11 & Castilla-La Mancha 19,84 (16) & 11 \\
\hline Extremadura …...... 7,69 (17) & 15 & Extremadura ......... $5,67(17)$ & $13 / 14$ \\
\hline
\end{tabular}

FUENTE: Esperanza de vida (Dopico y Reher, 1998, pp. 43-49); mortalidad infantil para 1900 (Dopico, 1985, p. 361) y para 1930 (Gómez Redondo, 1990, p. 276); alfabetización adulta en 1900 , calculada como número de personas alfabetizadas entre población mayor de diez años (Censo de la población de España... de 1900, Madrid, 1903, vol. I, pp. 477-479; y 1907, vol. III, pp. X, XIV, 323) y para 1930 (Núnez, 1992, pp. 132, 134, 163). El ranking PIB per capita relativo a partir de los datos de Álvarez Llano (1986, pp. 35, 37, 43) siguiendo el procedimiento de Carreras (1993, p. 20).

$(0,56)$, lo que, junto con las asimetrías observadas en la evolución del coeficiente de variación para uno y otro indicador, pone en tela de juicio la calidad de los cálculos efectuados en su día por Álvarez Llano (1986) si se quieren utilizar como indicadores indirectos del bienestar. Si se los supone aceptables como medidas de los medios y no del fin, habrá que reconocer, una vez más, que un producto per capita elevado en términos relativos no asegura necesariamente niveles más altos de bienestar, como muestran el caso de Madrid y Aragón hacia 1900 y, además de éstas, Valencia en 1930; y a la inversa, que con un producto per capita modesto en 
términos relativos se pueden conseguir niveles físicos de calidad de vida francamente buenos, como los de Baleares para 1900, o aceptables, como los de Galicia para esa fecha y 1930 .

\section{CONSIDERACIONES FINALES}

Nuestra pretensión con este trabajo ha sido llamar la atención sobre la utilidad de algunas técnicas y conceptos elaborados por la economía del desarrollo para definir más ampliamente y medir los niveles de vida del pasado, su grado de dispersión espacial y la tendencia que siguen en los momentos iniciales del crecimiento económico moderno de un país atrasado. Ello no sustituye en absoluto otro tipo de estudios que, siguiendo la clasificación gouldiana de los taxonomistas, o bien son «aglutinadores» (los estudios de magnitudes macroeconómicas agregadas a escala nacional) o bien son «separadores» de la realidad (los estudios de base local o regional que descienden hasta las economias familiares); más bien busca complementarlos. El énfasis creciente que unos y otros ponen en las estadísticas, y que este trabajo comparte, no debería hacernos olvidar el dictamen que el propio Gould (1981) realizó hace varios años a propósito de «la fascinación por los números» que invadió a la antropología en la segunda mitad del siglo Xxx y que después adoptó la psicología a principios del $\mathrm{XX}$, «abrumada por su reputación de ciencia "blanda"»: «gran parte de la fascinación de las estadísticas radica en nuestro sentimiento visceral de que las medidas abstractas que resumen amplios cuadros de datos tienen que expresar algo más real y más fundamental que los datos mismos».

Dicho de otra manera, la historia económica debe resistir el peligro de la reificación y algo tan complejo como el estudio del bienestar y la calidad de vida admite estrategias paralelas de investigación que no han hecho más que empezar. El IFCV es sólo una modesta contribución en esa línea de buscar la complementariedad. Pero las conclusiones que se extraen de la aplicación del IFCV para el caso de España entre 1860 y 1930 no nos parecen en absoluto limitadas: el aumento de las disparidades territoriales de los niveles de vida que acompañaron a los primeros momentos del crecimiento económico moderno en España no fue impedimento para que se produjera una mejora, desigual, pero generalizada de los mismos (y superior a la experimentada en términos de producto per capita); las semejanzas con el caso italiano de transición al desarrollo se confirman por nuevas vías tanto en este punto como en la distribución geográfica 
de los resultados y la influencia en los mismos de las estructuras agrarias; por último, las disparidades espaciales del bienestar presentan importantes diferencias en comparación con las que arrojan las estimaciones de renta regional per capita en el mismo periodo, lo que matiza las cifras corrientemente utilizadas y sugiere la necesidad de tener una visión más cautelosa de la relación entre crecimiento económico, tal y como habitualmente suele medirse, y bienestar en términos de resultados para la salud y la educación de la población.

\section{ANEXO 1 \\ Fuentes para la construcción del IFCV a nivel provincial}

Los datos de mortalidad infantil para 1860 están calculados en tantos por 1.000 sobre la media de las defunciones de menores de 1 año para el período 1859-1861 y la población menor de un año del Censo de 1860 (están tomados del Anuario estadístico de España correspondiente a 1859-1860, Madrid, 1860, pp. 132, 142; Anuario estadístico de España correspondiente a 1860-1861, Madrid, 1862-1863, pp. 50, 56, 62, 68, y Censo de la población... de 1860, Madrid, 1863, pp. 715-716). A pesar de que estrictamente hablando no es la tasa de mortalidad infantil, el cociente resultante se denomina (y usa internacionalmente como) tasa de mortalidad infantil y expresa la probabilidad de muerte a los 0 años de edad. Las cifras en las que se basa han sido duramente criticadas en razón de que su origen todavía eclesiástico provocó un importante subregistro de las defunciones de recién nacidos (que no se incluirian en las estadísticas hasta 1930) y la acumulación de errores en el trasvase de las estadísticas desde los párrocos a los ayuntamientos y de éstos a los partidos judiciales, como han señalado Dopico (1985), Livi-Bacci (1993) y Gómez Redondo (1992). Esto es obvio si se atiende a la comparación entre las tasas de mortalidad infantil de 1861-1865 y las tablas de supervivencia de 1863-1870 (en cuya construcción se elude el subregistro al considerar sólo a la población mayor de seis años), pero sobre todo resalta en dos regiones, Galicia y Asturias, que se encuentran entre las que presentan unos coeficientes de población rural más altos, según los datos elaborados por Gómez Mendoza y Luna (1986). En ambas regiones, la mortalidad infantil, deducida a partir de los datos de Dopico (1987) como 1.000 menos la tasa de supervivencia a la edad de 1 año para el período 1863-1870, supone respectivamente un 86 y un 117 por 100 más que la tasa de mortalidad infantil de 1861-1865, 
sobre una diferencia para el caso de España del 31 por 100 entre los dos cálculos. En vista de lo cual se han rectificado los datos de Oviedo $(102,47)$ y Pontevedra $(110,56)$, las dos únicas provincias de España en las que nuestra tasa de mortalidad infantil de 1859-1861 es más baja que la de 1901-1905. Para ello hemos procedido a sustituir las cifras originales mediante el expeditivo procedimiento de asignarles los respectivos porcentajes de incremento de Asturias y Galicia. Por su parte, los datos de mortalidad infantil de 1911-1915 y 1931-1935 proceden de Gómez Redondo (1992) y no incluyen la corrección del subregistro de los muertos al nacer, si bien la autora considera para fechas posteriores que ello supuso no más allá de un 5 por 100 del total con una «tendencia a la estabilidad [que] es muy probable que se prolongase en el pasado».

En cuanto a la esperanza de vida, se han utilizado para 1860 las estimaciones de Dopico (1987) a partir de tablas de mortalidad siguiendo el modelo de Coale y Demeny (Livi-Bacci 1991). Según Dopico (1987), los resultados para las provincias gallegas y Asturias son poco fiables y sólo dos provincias, Santander y Baleares, siguen el modelo occidental, mientras que el resto de España corresponde al modelo del sur; este trabajo pionero no ofrece estimaciones para Canarias por la mala calidad de los datos, por lo que se ha interpolado una cifra proyectando hacia atrás la recta de tendencia de la esperanza de vida a partir de los datos de esa provincia en 1930,1920, 1910 y 1900 (estos datos, como los del resto de España para 1900, 1910 y 1930 también pertenecen a las estimaciones de Dopico y Reher, 1998).

Finalmente, los datos de alfabetización de 1860, 1900, 1910 y 1930 se han tomado del trabajo de Núñez (1992) y expresan una tasa porcentual entre población alfabetizada (que sabe leer y escribir) y la población mayor de diez años. La fiabilidad de las fuentes en las que se basan parece bastante aceptable (con una ocultación de pequeña magnitud y constante a lo largo del tiempo) y, aunque se ha criticado el peculiar procedimiento de construcción de la tasa porque «sobrestima en nivel de alfabetización general», parece que «otras tasas calculadas con distinto criterio a partir de las mismas fuentes muestran un idéntico panorama general y la misma distribución regional del fenómeno» (Pérez Moreda 1997). 


\section{ANEXO 2}

IFCV dinámico en España a nivel provincial, 1860-1930 y tasas medidas de reducción de la disparidad

\begin{tabular}{|c|c|c|c|c|c|c|c|c|}
\hline Provincias & $\begin{array}{l}I F C V \\
1860\end{array}$ & $\begin{array}{c}I F C V \\
1900\end{array}$ & $\begin{array}{r}\text { IFCV } \\
1910\end{array}$ & $\begin{array}{c}I F C V \\
1930\end{array}$ & $1860-1930$ & $1860-00$ & $1900-30$ & $1910-30$ \\
\hline Álava. & 42,89 & 65,43 & 76,29 & 92,04 & 1,10 & 1,06 & 1,14 & 0,94 \\
\hline Albacete. & 23,50 & 37,38 & 44,92 & 64,79 & 1,46 & 1,17 & 1,85 & 1,85 \\
\hline Alicante.. & 25,31 & 48,76 & 56,56 & 75,20 & 1,57 & 1,65 & 1,45 & 1,43 \\
\hline Almería. & 24,20 & 33,20 & 46,37 & 72,95 & 1,59 & 0,79 & 2,66 & 2,29 \\
\hline Ávila....... & 21,19 & 44,87 & 54,52 & 72,24 & 1,77 & 1,89 & 1,60 & 1,42 \\
\hline Badajoz. & 20,24 & 36,81 & 52,62 & 68,87 & 1,76 & 1,51 & 2,11 & 1,35 \\
\hline Baleares ................. & 40,11 & 57,83 & 68,09 & 86,52 & 1,10 & 0,92 & 1,35 & 1,20 \\
\hline Barcelona.... & 27,99 & 56,47 & 67,90 & 88,91 & 1,66 & 1,77 & 1,52 & 1,36 \\
\hline Burgos .................... & 35,41 & 55,01 & 65,84 & 80,05 & 1,17 & 1,11 & 1,26 & 0,98 \\
\hline Cáceres ............... & 17,21 & 39,07 & 46,77 & 61,68 & 1,84 & 2,07 & 1,53 & 1,39 \\
\hline Cádiz .................... & 25,48 & 43,13 & 52,21 & 69,49 & 1,44 & 1,32 & 1,60 & 1,44 \\
\hline 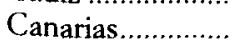 & 17,51 & 50,20 & 52,79 & 70,32 & 2,01 & 2,67 & 1,13 & 1,44 \\
\hline Castellón.............. & 21,54 & 46,37 & 57,45 & 76,65 & 1,83 & 1,94 & 1,69 & 1,45 \\
\hline Ciudad Real......... & 19,87 & 40,97 & 47,50 & 66,64 & 1,74 & 1,83 & 1,63 & 1,71 \\
\hline Córdoba .............. & 24,52 & 36,49 & 49,74 & 70,14 & 1,51 & 1,00 & 2,20 & 1,73 \\
\hline Coruña (La)........ & 38,56 & 52,94 & 62,31 & 77,52 & 1,00 & 0,80 & 1,28 & 1,10 \\
\hline Cuenca .................. & 21,81 & 42,77 & 50,39 & 68,18 & 1,64 & 1,70 & 1,57 & 1,52 \\
\hline Gerona... & 26,58 & 52,32 & 68,71 & 90,27 & 1,76 & 1,71 & 1,83 & 1,37 \\
\hline Granada.............. & 23,51 & 35,62 & 47,49 & 73,15 & 1,64 & 1,04 & 2,43 & 2,18 \\
\hline Guadalajara ......... & 25,50 & 48,35 & 59,04 & 77,98 & 1,61 & 1,61 & 1,61 & 1,40 \\
\hline Guipúzcoa .......... & 40,01 & 62,80 & 76,65 & 87,75 & 1,13 & 1,13 & 1,12 & 0,68 \\
\hline Huelva ........ & 31,21 & 49,55 & 59,24 & 75,14 & 1,26 & 1,16 & 1,40 & 1,20 \\
\hline Huesca... & 25,50 & 50,90 & 58,81 & 82,17 & 1,69 & 1,74 & 1,61 & 1,69 \\
\hline Jaén........ & 24,24 & 31,21 & 42,48 & 65,37 & 1,43 & 0,63 & 2,50 & 2,18 \\
\hline León .. & 34,60 & 57,21 & 64,26 & 81,38 & 1,23 & 1,27 & 1,18 & 1,19 \\
\hline Lérida .................... & 21,27 & 50,92 & 64,54 & 89,87 & 2,08 & 2,21 & 1,91 & 1,67 \\
\hline Logroño ............... & 25,34 & 52,77 & 65,25 & 81,56 & 1,68 & 1,85 & 1,46 & 1,12 \\
\hline Lugo ........................ & 34,93 & 53,65 & 61,95 & 79,86 & 1,19 & 1,08 & 1,33 & 1,28 \\
\hline Madrid... & 15,17 & 53,21 & 64,47 & 82,33 & 2,45 & 3,19 & 1,47 & 1,23 \\
\hline Málaga .................. & 25,11 & 37,93 & 46,30 & 73,02 & 1,54 & 1,04 & 2,21 & 2,30 \\
\hline 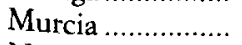 & 24,37 & 39,08 & 46,20 & 70,73 & 1,53 & 1,19 & 2,00 & 2,15 \\
\hline Navarra................ & 37,74 & 60,99 & 74,30 & 88,00 & 1,22 & 1,21 & 1,23 & 0,85 \\
\hline Orense.... & 34,55 & 46,14 & 58,84 & 76,91 & 1,15 & 0,73 & 1,72 & 1,35 \\
\hline Oviedo.... & 40,74 & 59,38 & 72,25 & 87,87 & 1,10 & 0,95 & 1,31 & 0,98 \\
\hline Palencia. & 25,91 & 52,49 & 61,83 & 75,83 & 1,55 & 1,78 & 1,23 & 1,03 \\
\hline Pontevedra . & 39,78 & 58,75 & 66,39 & 80,92 & 1,02 & 0,98 & 1,07 & 0,99 \\
\hline 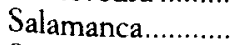 & 30,79 & 56,33 & 63,66 & 80,31 & 1,38 & 1,52 & 1,19 & 1,17 \\
\hline 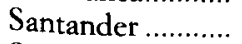 & 45,89 & 65,97 & 82,09 & 89,53 & 0,96 & 0,91 & 1,02 & 0,43 \\
\hline Segovia ..... & 24,59 & 55,83 & 65,85 & 83,10 & 1,75 & 2,07 & 1,33 & 1,17 \\
\hline 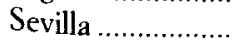 & 27,84 & 41,37 & 49,63 & 72,24 & 1,37 & 0,99 & 1,88 & 1,89 \\
\hline
\end{tabular}




\begin{tabular}{|c|c|c|c|c|c|c|c|c|}
\hline Provincias & $\begin{array}{c}I F C V \\
1860\end{array}$ & $\begin{array}{c}I F C V \\
1900\end{array}$ & $\begin{array}{l}\text { IFCV } \\
1910\end{array}$ & $\begin{array}{c}I F C V \\
1930\end{array}$ & $1860-1930$ & $1860-00$ & $1900-30$ & $1910-30$ \\
\hline Soria & 28,86 & 40,74 & 65,31 & 83,20 & 1,52 & 0,87 & 2,41 & 1,22 \\
\hline Tarragona. & 32,80 & 55,83 & 67,24 & 89,48 & 1,44 & 1,34 & 1,58 & 1,44 \\
\hline Teruel ................... & 23,50 & 44,18 & 54,86 & 76,14 & 1,69 & 1,59 & 1,83 & 1,65 \\
\hline Toledo ....... & 23,13 & 42,90 & 53,61 & 72,87 & 1,65 & 1,56 & 1,78 & 1,55 \\
\hline Valencia.. & 18,85 & 45,97 & 58,89 & 77,02 & 2,03 & 2,25 & 1,74 & 1,35 \\
\hline Valladolid ............ & 22,58 & 47,74 & 61,01 & 78,78 & 1,80 & 1,89 & 1,68 & 1,29 \\
\hline 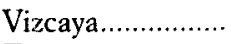 & 45,29 & 59,11 & 75,91 & 90,54 & 0,99 & 0,67 & 1,43 & 0,89 \\
\hline Zamora ................ & 23,65 & 53,48 & 61,23 & 75,51 & 1,67 & 2,06 & 1,16 & 1,05 \\
\hline Zaragoza ............... & 16,90 & 41,84 & 55,68 & 77,69 & 2,20 & 2,29 & 2,08 & 1,68 \\
\hline ESPANA ............ & 27,85 & 47,73 & 58,72 & 77,71 & 1,48 & 1,36 & 1,64 & 1,41 \\
\hline
\end{tabular}

\section{BIBLIOGRAFÍA}

Adelman, I., y MoRrIS, C. T. (1965): «A factor analysis of the interrelationship between social and political variables and per capita Gross National Product», Quarterly Journal of Economics, 79, pp. 555-578.

Álvarez LlANo, R. (1986): «Evolución de la estructura económica regional de España en la historia: Una aproximación», Situación, 1, pp. 5-61.

BaIroch, P. (1981): «The Main Trends in National Economic Disparities since the Industrial Revolution», en BAIROCH, P., y LÉVY-LEBOYER, M. (eds.), pp. 3-17.

- y LeVY-Leboyer, M. (eds.) (1981): Disparities in Economic Development since the Industrial Revolution, Londres, MacMillan.

BANCO DE BILBAO (1978): Renta Nacional de España y su distribución provincial, serie bomogénea, 1955-1975, Bilbao, Banco Bilbao.

BardinI, C.; CARreraS, A., y Lains, P. (1995): «The National Accounts for Italy, Spain and Portugal», Scandinavian Economic History Review, 53 (1), pp. 115-146.

Beckerman, W. (1993): «Is Economic Growth Still Desirable?», en Szimal, A.; VAN ARK, B., y Pilat, D. (eds.), pp. 77-100.

Bustelo, F. (1993): «Los cálculos del Producto Nacional en los siglos xIX y xx y su utilización en la historia económica», Revista de Historia Económica, 11 (1), pp. 155-177.

CARRERAS, A. (1993): «Fuentes y datos para el análisis regional de la industrialización española», en Nadal, J., y Carreras, A. (eds.): Pautas regionales de la industrialización española (siglos XIX y XX), Barcelona, Ariel, pp. 3-20.

CARRIÓN, P. (1973): La reforma agraria de la segunda república y la situación actual de la agricultura española, Barcelona, Ariel.

Cipolla, C. M. (1970): Educación y desarrollo en Occidente, Barcelona, Ariel.

CrAfTS, N. F. R. (1984): «Patterns of development in nineteenth century Europe», Oxford Economic Papers, 36, pp. 438-458.

- (1993): «Was the Thatcher Experiment Worth It? British Economic Growth in a European Context», en Szimai, A.; VAN ARK, B., y Pilat, D. (eds.), pp. $327-350$. 
- (1997): «Some dimensions of "quality of life" during the British industrial revolution», Economic History Review, 50 (4), pp. 617-639.

Dasgupta, P., y Weale, M. (1992): «On Measuring de Quality of Life», World Development, 20 (2), pp. 119-131.

DESAI, M. (1993): «Income and alternative measures of well-being», en WESTENDORFF, D., y Ghai, D. (eds.), pp. 23-39.

Devolder, D. (s.f.): «Tablas de mortalidad provinciales», en Centre d'Estudis Demogràfics, Papers de Demografia, 15.

DopICo, F. (1985): «Desarrollo económico y social y mortalidad infantil. Diferencias regionales (1900-1950)», en Asociación Española de Ciencia Regional, Crisis, autonomia y desarrollo regional. IX Reunion de Estudios Regionales, Santiago, I, pp. 358-372.

- (1987): «Regional Mortality Tables for Spain in the 1860s», Historical Methods, $20(4)$, pp. $173-179$.

DOPico, F., y ReHER, D. S. (1998): El declive de la mortalidad en España, monografía 1 de la $\mathrm{ADEH}$.

Federico, G., y Toniolo, G. (1991): «Italy», en Sylla, R., y Toniolo, G. (eds.), pp. 197-217.

FonTANA, J. (1990): «Nivel de vida, calidad de vida: un intento de estado de la cuestión y algunas reflexiones», en XV Simpsi d'Anàlisis Econòmica, Barcelona, I, pp. 1-13.

GalleGO, D. (1993): «Pautas regionales de cambio técnico en el sector agrario español (1900-1930)», Cuadernos Aragoneses de Economia, 3 (2), pp. 241-276.

Gómez MendozA, A., y Luna, G. (1986): «El desarrollo urbano en España, 1860-1930», Boletín de la Asociación de Demografía Histórica, 4 (2), pp. 3-22.

Gómez Redondo, R. (1992): La mortalidad infantil española en el siglo XX, Madrid, CIS.

GOOD, D. A. (1981): «Economic Integration and Regional Development in Austria-Hungary, 1867-1913», en Bairoch, P., y LÉVY-Leboyer, M. (eds.), pp. 137-150.

-- (1991): «Austria-Hungary», en Sylla, R., y Toniolo, G. (eds.), pp. 218-27.

Gould, S. J. [(1981) 1986]: La falsa medida del bombre, Barcelona, Orbis.

Haines, M. R. (1985): «Income and Chilhood Mortality: A Comparison of England and Wales, 1911, and the United States, 1900», Journal of Economic History, 44 (4), pp. 885-912.

Hicks, N., y Streeten, P. (1979): «Indicators of Development: The Search for a Basic Needs Yardistick», World Development, 7 (6), pp. 567-580.

LARSON, D. A., y WILFORD, W. T. (1979): «The Physical Quality of Life Index: A Useful Social Indicator?», World Development, 7 (6), pp. 581-584.

Liv-BACCI, M. (1990): Historia mínima de la población mundial, Barcelona, Ariel.

- (1993): Introducción a la demografía, Barcelona, Ariel.

LUNA, G. (1988): «La población urbana en España, 1860-1930», Boletín de la Asociación de Demografía Histórica, 6 (1), pp. 25.71.

MadDIsOn, A. (1991): Historia del desarrollo capitalista. Sus fuerzas dinámicas, Barcelona.

MALEFAKIS, E. (1980): Reforma agraria y revolución campesina en la España del siglo $\mathrm{XX}$, Barcelona, Ariel. 
MiKelaRENA, F. (1993): «Los movimientos migratorios interprovinciales en España entre 1877 y 1930: áreas de atracción, áreas de expulsión, periodización cronológica y cuencas migratorias», Cuadernos Aragoneses de Economía, 3 (2), pp. 213-240.

Mironov, B. N. (1993): «Educación y desarrollo económico en Rusia, siglos XIX y xX», en NúNEZ, C. E., y TORTELlA, G. (eds.), pp. 271-306.

MORRIS, M. D. (1979): Measuring the Condition of the World's Poor: The Physical Quality of Life Index, New York, Overseas Development Council.

- y McAlpIN, M. B. (1982): Measuring the Condition of India's Poor. The Physical Quality of Life, New Delhi, Promilla\&CO.

Murray, C. J. L. (1993): «Development data constraints and the human development index», en WESTENDORFF, D., y GHAI, D. (eds.), pp. 40-64.

NúNEZ, C. E. (1992): La fuente de la riqueza. Educación y desarrollo económico en la España contemporánea, Madrid, Alianza.

- (1993): «Educación y desarrollo en el continente americano», en NuNNEZ, C. E., y TORTElla, G. (eds.), pp. 359-380.

- y TORTElla (eds.) (1993): La maldición divina. Ignorancia y atraso económico en perspectiva bistórica, Madrid, Alianza.

OTHICK, J. (1983): «Development Indicators and the Historical Study of Human Welfare: Towards a New Perspective», Joumal of Economic History, 43 (1), pp. 63-70.

Pérez. MoReda, V. (1997): «El proceso de alfabetización y la formación de capital humano en España», Papeles de Economia Española, 73, pp. 243-253.

PNuD (1990): «Definición y medición del desarrollo humano», Desarrollo, 17, pp. 8-12.

Prados, P. (1992): «La historia económica de España e Italia: introducción», en Prados, L., y Zamagni, V. (eds.): El desarrollo económico en la Europa del Sur: España e Italia en perspectiva bistórica, Madrid, Alianza, pp. 27-55.

- (1993): «Long-run Economic Growth in Spain since 1800: An International Perspective», en Szimal, A.; Van ArK, B., y Pilat, D. (eds.), pp. 267-284.

Rowley, C. K. (1987): «Borda, Jean-Charles de», en Eatwell, J.; Milgate, M., y Newman, P. (eds.): The New Palgrave. A Dictionary of Economics, vol. I, London, CUP, pp. 262-263.

Sánchez. Alonso, B. (1995): Las causas de la emigración española, 1880-1930, Madrid, Alianza.

SEN, A. K. (1981): «Public action and the quality of life in Developing Countries», Oxford Bulletin of Economics and Statistics, 43 (4), pp. 287-319.

- (1984): Resources, Values and Development, Cambridge (Mass.), Harvard Univ. Press.

- et al. (1987): The Standard of Living, Cambridge, CUP.

- (1988): «The Concept of Development», en Chenery, H., y SRInivasan, T. N. (eds.): Handbook of Development Economics, vol. I, Amsterdad, New Holland, pp. 9-26.

- (1993): «Capability and Well-Being», en Nussbaum, M., y SEN, A. (eds.): The Quality of Life, Oxford, pp. 30-53.

- (1995): Inequality reexamined, Oxford, Clarendon Press. 
Silber, J. (1983): «ELL (The Equivalente Length of Life) or Another Attempt at Measuring Development», World Development, 11 (1), pp. 21-29.

SIMPSON, J. (1994): «La producción y la productividad agraria españolas, 1890-1936», Revista de Historia Económica, 12 (1), pp. 43-84.

- (1997a): «Economic Development in Spain, 1850-1936», Economic History Review, 50 (2), pp. 348-359.

- (1997b): La agricultura española (1765-1965): la larga siesta, Madrid, Alianza.

Silla, R, y Toniolo, G. (eds.) (1991): Patterns of European Industrialization. The Nineteenth Century, London, Routledge.

SzIMAI, A.; VAN ARK, B., y PILAT, D. (eds.) (1993): Explaining Economic Growth. Essays in Honour of Agnus Maddison, Amsterdam, Elsevier Science Publishers.

Todaro, M. P. (1988): El desarrollo económico del Tercer Mundo, Madrid, Alianza.

Toniolo, G. (1988): Storia economica dell'Italia liberale (1850-1918), Bologna, Il Mulino.

TORTEllA, G. (1994): «Patterns of economic retardation and recovery in south-western Europe in the nineteenth and twentieth centuries», Economic History Review, 47 (1), pp. 1-21.

Toutain, J. C. (1981): «The Uneven Growth of Regional Incomes in France from 1840 to 1970 », en Bairoch, P., y Lévy-Leboyer, M. (eds.), pp. 302.315.

USHER, D. (1973): «An Imputation to the Measure of Economic Growth for Changes in Life Expectancy», en Moss, M. (ed.): The Measurement of Economic and Social Performance, New York, Columbia Univ. Press, pp. 93-226.

VALLIN, J. (1989): «Le mortalité en Europe de 1720 à 1914: tendances à long terme et changements de structure par sexe et par age», Annales de Démographie Historique, pp. 31.54.

WestendorfF, D., y GhaI, D. (eds.) (1993): Monitoring Social Progress in the 1990s. Data constraints, concerns and priorities, Aldershot, Avebury.

Williamson, J. G. (1965): «Regional inequality and the process of national development: A description of the patterns», Economic Devlopment and Cultural Change, 13 (4-2), pp. 3-45.

- (1981): «Inequality and Regional Development: the View from America», en Bairoch, P., y LEVY-LeboYer, M. (eds.), pp. 373-391.

- (1991): Inequality, Poverty and History, Cambridge (Mass.), Blackwell.

Woons, R. I. (1993): «On the Historical Relationship Between Infant and Adult Mortality», Population Studies, 47 (2), pp. 195-219.

- Watterson, P. A., y WoOdward, J. H. (1988): «The Causes of Rapid Infant Mortality Decline in England and Wales, 1861-1921. Part I», Population Studies, 42, pp. 343-366.

ZAMAGNI, V. (1989): «An international comparison of real wages, 1890-1913: Methodological issues and results», en ScHolliers, P. (ed.): Real wages in 19th and 20 th century Europe. Historical and comparative perspectives, Oxford, Berg, pp. 107-139.

- (1993): «Instrucción y desarrollo económico en Italia, 1861-1913», en NúNEZ, C. E., y Tortella, G. (eds.), pp. 181-222. 